Como citar este artigo:

Boff JM, Strasburg VJ. Avaliação da efetividade de boas práticas em serviços de alimentação coletiva em uma capital brasileira. Revista Saúde (Sta. Maria). 2018; 44(1): 1-7

Autor correspondente: Roberta Lamonatto Taglietti E-mail: rotagli@unochapeco.edu. br

Telefone: (49) 988095539

Formação Profissional:

Nutricionista, mestra e doutoranda em Ciências da Saúde, pela

Universidade Comunitária

da Região de Chapecó

(Unochapecó), Chapecó, Santa

Catarina (SC), Brasil.

Filiação Institucional: Universidade Comunitária da Região de

Chapecó (Unochapecó)

Endereco para correspondencia:

Rua: Av. Senador Atílio Fontana

$n^{\circ}: 591$ Bairro: Efapi

Cidade: Chapecó Estado: SC

CEP: 89809-000.

Data de Submissão: 08/11/2017

Data de aceite: 15/03/2018

Conflito de Interesse: Não há

conflito de interesse

\section{Avaliação dos aspectos relacionados às escolhas alimentares entre professores de centros educacionais infantis}

\section{Evaluation of aspects related to food choices between teachers of children educational centers}

\author{
Roberta Lamonatto Taglietti, Aline Cristina Lalana, Talita Menezes da Rosa, \\ Carla Rosane Paz Arruda Teo, Fernanda Grison Confortin
}

\section{RESUMO}

Objetivo: Avaliar aspectos relacionados às escolhas alimentares e consumo alimentar de professores de Centros Educacionais Infantis de um município do Estado de Santa Catarina. Método: Realizouse um estudo transversal descritivo quantitativo, do qual participaram 100 professores, respondendo a questionários sobre o motivo de suas escolhas alimentares e consumo alimentar. Resultados: 0 fator apelo sensorial foi atribuído com maior relevância para determinar as escolhas alimentares dos professores, seguido dos fatores saúde, conteúdo natural e controle de peso, associados com requisitos importantes para promoção de hábitos alimentares saudáveis. Os participantes consumiram com maior frequência alimentos in natura e minimamente processados. Conclusão: Os resultados reforçam que os professores podem ser influências positivas na formação de hábitos alimentares de crianças, mas ainda é preciso conscientizá-los sobre essa responsabilidade, preparando-os para abordar a alimentação na infância, especialmente em um momento em que se observa diminuição do compartilhamento familiar das refeições.

Descritores: Professor; Hábitos Alimentares; Educação Infantil.

\begin{abstract}
Objective: to evaluate aspects related to food choices and food consumption of teachers of Child Educational Centers of a municipality in Santa Catarina state. Method: a quantitative descriptive cross-sectional study was carried out, in which 100 teachers participated, responding to questionnaires about the reason for their food choices and food consumption. Results: the sensory appeal factor was attributed with greater relevance to determine the teachers' food choices, followed by health, natural content and weight control factors, associated with important requirements to promote healthy eating habits. The participants consumed more often in natura and minimally processed foods. Conclusion: the results reinforce that teachers can be positive influences in the formation of children's eating habits, but it is still necessary to make them aware of this responsibility, preparing them for approaching feeding in childhood, especially at a time when there is a decrease in family sharing of meals.
\end{abstract}

Keyword: Faculty; Feeding Behavior; Child Rearing. 


\section{Introdução}

Aquilo que se come e bebe não é somente uma questão de escolha individual, existem outros fatores como sociais, econômicos e ambientais que podem restringir as escolhas alimentares. Em grande parte, o que se consome, envolve questões familiares e sociais ${ }^{1}$. No entanto, mesmo o homem sendo capaz de consumir biologicamente de tudo, as escolhas alimentares se baseiam nos sistemas culturais dos grupos humanos, os quais só se permitem alimentar-se do que é aceito culturalmente? ${ }^{2}$.

Neste sentido, é preciso trazer para discussão o período da infância, sendo este marcado pela formação de hábitos e comportamentos alimentares, promovido por meio da interação da criança com o alimento, pelo seu desenvolvimento anatomo-fisiológico e por fatores emocionais, psicológicos, socioeconômicos e culturais. Vale destacar que o mais relevante de todos é a influência da própria família e de pessoas ligadas à sua alimentação ${ }^{3}$.

É preciso compreender que experiências precoces e a interação constante com os alimentos na infância, podem determinar hábitos e preferências quando adultos ${ }^{4}$. Em um primeiro momento, a alimentação é influenciada pelos pais e em seguida relaciona-se com o ambiente no qual a criança está inserida, como a escola, tendo como referência os professores ${ }^{5}$

Neste contexto, é preciso chamar a atenção para a importância da escola neste processo, pois é onde a criança começa a estabelecer novas relações com o mundo, conhece novas realidades e firma laços de amizade com professores e colegas, acostumando-se a um novo ambiente6. Além disso, o educador é o colaborador responsável por auxiliar a criança durante a alimentação, especialmente nos primeiros anos escolares, refletindo seu papel primordial na constituição dos hábitos alimentares ${ }^{7}$.

Vale destacar que o consumo dos alimentos ultraprocessados, de alta densidade energética e com adição de açúcar, se correlacionna com as taxas de excesso de peso, obesidade e distúrbios metabólicos que têm marcado 0 quadro epidemiológico e nutricional do País. Dados apontam que uma em cada três crianças apresenta excesso de peso, sendo $18,9 \%$ da população obesa, demonstrando que a obesidade cresceu $60 \%$ em 10 anos ${ }^{8-10}$.

Segundo registro do Sistema de Vigilância Alimentar e Nutricional (SISVAN), no ano de 2016, 15,04\% e 28,53\% das crianças brasileiras com idade entre zero e cinco e cinco a dez anos apresentavam excesso de peso (sobrepeso e obesidade respectivamente). Para a mesma população da Região Sul do Brasil e do Estado de Santa Catarina, o sobrepeso e a obesidade representam $13,91 \%$ e $34,46 \%, 11,94 \%$ e 32,5\% respectivamente 11 . Este é o cenário de um processo denominado transição nutricional, caracterizado por queda das taxas de desnutrição, aumento da prevalência de obesidade e incremento de casos de "fome oculta", representada por deficiências nutricionais específicas ${ }^{12}$.

Nesta ótica, a escola é considerada um espaço privilegiado para práticas de promoção de saúde, incluindo ações de educação alimentar e nutricional ${ }^{13}$. Neste contexto escolar, o educador tem papel muito importante, por ser ele 0 facilitador/mediador do conhecimento ${ }^{14}$.

Tendo em vista que o professor tem posicionamento estratégico no âmbito escolar e exerce influência sobre as escolhas e preferências alimentares dos educandos, o objetivo deste trabalho é avaliar aspectos relacionados às escolhas alimentares de professores de Centros Educacionais Infantis de um Município do Estado de Santa Catarina (SC).

\section{Metodologia}

Este estudo possui delineamento transversal descritivo com abordagem quantitativa. Foi realizado em um Município de Santa Catarina, e contou com a participação de 100 professores de oito Centros Educacionais Infantis Municipais (CEIM) indicados pela Secretaria Municipal de Educação (SEDUC). Assumiu-se a opção de entrevistar todos os professores presentes no momento da abordagem em cada CEIM e que concordaram em responder aos instrumentos da pesquisa.

Como instrumento para coleta de informações foi utilizado um questionário autoaplicável sobre motivos das escolhas alimentares (Food Choice Questionnaire- FCQ)15, desenvolvido originalmente na Língua Inglesa. 0 questionário é composto por 36 itens, distribuídos em nove fatores: saúde, humor, conveniência, apelo sensorial, conteúdo natural, preço, controle de peso, familiaridade e preocupação ética, adaptado para língua portuguesa16. Seguindo este instrumento, 
primeiramente, o participante é convidado a refletir sobre a declaração "Para mim é importante que o alimen-to que eu coma no dia a dia seja...", indicando, em seguida, dentro de cada fator, os itens que refletem o que considera ser mais importante para determinar sua escolha alimentar. As opções de resposta foram apresentadas em uma escala de avaliação do tipo likert, variando de 1 a 4 pontos: 1 (nada im ᄀportante); 2 (um pouco importante); 3 (mode rradamente importante); e 4 (muito importante). Dessa forma, gerou-se um escore17.

Complementar a este instrumento, foi utilizado um formulário para avaliação de marcadores de consumo alimentar estabelecido pelo Ministério da Saúde (MS), que propõe a avaliação de alimentos consumidos no dia anterior, o que ameniza possíveis vieses de memória18. A aplicação deste questionário ocorreu no mesmo dia da aplicação do FCQ.

Para análise e interpretação dos resultados do questionário $F C Q$ e do formulário de marcadores do consumo alimentar, utilizou-se estatística descritiva. No que concerne à pontuação do $\mathrm{FCQ}$, foram consideradas as respostas com escores maiores, sendo assim, pontuações mais eleva 7 das indicaram que o participante atribuiu maior importância a determinado fator ${ }^{16}$. As pontuações em cada uma das nove dimensões do FCQ foram computadas com média de classificações de itens por dimensão.

Os resultados obtidos através do formulário de marcadores do consumo alimentar18, foram expressos por meio da frequência relativa e absoluta da população e avaliados a partir de recomendações estabelecidas pelo Guia Alimentar para a População Brasileira? .

O projeto foi aprovado pelo Comitê de Ética em Pesquisa Envolvendo Seres Humanos (CEP), sob o parecer $n^{0} 1.480 .788$ de 06/04/2016, sendo respeitados todos os preceitos éticos para a pesquisa evolvendo seres humanos dispostos na resolução $n^{0} 466 / 2012$. Todos os professores aceitaram participar da pesquisa e autorizaram a utilização das informações mediante assinatura do termo de consentimento livre e esclarecido.

\section{RESULTADOS E DISCUSSÃO:}

A amostra deste estudo foi constituída por indivíduos com faixa etária entre 22 a 52 anos (média de 35 anos) e predominância do sexo feminino $(93 \% ; n=93)$. Em relação ao tempo de atuação na função, destaca-se que houve variação de 2 meses a 28 anos. Quanto à formação acadêmica, 61\% $(n=61)$ possuem pós-graduação em nível de especialização e mestrado.

Com relação aos resultados obtidos com a aplicação do questionário FCQ (Figura 1), é possível observar que o fator apelo sensorial foi atribuído com maior importância para determinar as escolhas alimentares dos professores. É importante mencionar que este fator está relacionado com as características sensoriais dos alimentos, como: cor, sabor, textura e odor.

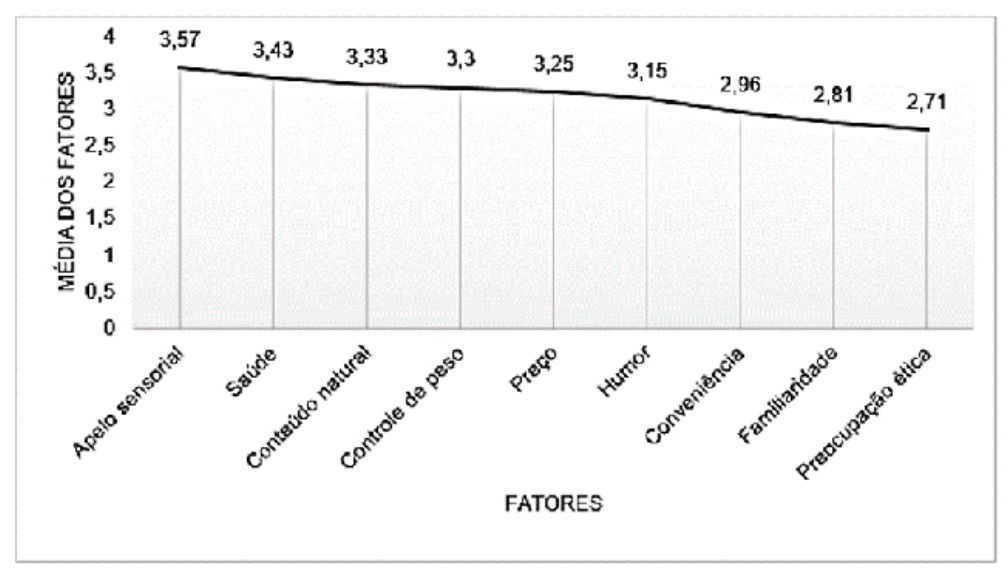

Г qura 1. Médie de fatores sobre o motivo des escolhes alimentares de professores de Centros Educacionais Infants de um Munićpio de Senta Cetarine 2010. 
AVALIAÇÃO DOS ASPECTOS RELACIONADOS ÀS ESCOLHAS ALIMENTARES ENTRE PROFESSORES DE CENTROS EDUCACIONAIS INFANTIS

Figura 1: Média de fatores sobre o motivo das escolhas alimentares de professores de Centros Educacionais Infantis de um Município de Santa Catarina, 2016.

Destaca-se que, neste estudo, quando discutido o apelo sensorial, os participantes referiram-se a características que lembram a comida de verdade, sem aditivos químicos e conservantes, ou seja, alimentos in natura ou minimamente processados. Resultado semelhante a nossa pesquisa foi evidenciado em um estudo realizado com consumidores na Bélgica, Hungria, Romênia e Filipinas, onde participaram 1420 consumidores. Esse estudo demonstrou que todos os participantes europeus também consideram o fator apelo sensorial como o mais importante na escolha diária de alimentos. A saúde, a conveniência e o preço também foram fatores importantes para as escolhas alimentares na Bélgica, na Hungria e na Romênia. Para os filipinos, os mais importantes foram a saúde, o preço e o humor19.

$\mathrm{O}$ apelo sensorial é o fator mais importante entre todos os consumidores europeus, enquanto a saúde, a conveniência e o preço foram todos os cinco fatores mais importantes que definem a escolha de alimentos na Bélgica, na Hungria e na Romênia. Para os filipinos, os mais importantes foram a saúde, o preço e o humor.

É preciso destacar que os quatro primeiros fatores indicados como os mais representativos nas escolhas alimentares dos professores - apelo sensorial, saúde, conteúdo natural e controle de peso - estão associados com requisitos importantes para promoção de hábitos alimentares saudáveis. Diante disso, considera-se este resultado positivo, pois o professor nortear suas escolhas alimentares a partir destes fatores é um bom indicador de que pode reproduzir as motivações no ambiente escolar, onde é visto como exemplo e referência pelos escolares, influenciando diretamente na formação de hábitos alimentares saudáveis e nas escolhas alimentares dos mesmos.

Sabe-se que a escola é um espaço fundamental para a saúde dos pré-escolares. Nesse sentido, o ambiente escolar deve ser um espaço dinâmico, com relações interativas, no qual o prazer, a criatividade e o desenvolvimento da autonomia necessitam estar presentes. Deste modo, a escola contribui para o desenvolvimento de maiores habilidades de escolha dos pré-escolares para uma vida mais saudável ${ }^{20}$.

O ambiente escolar tem papel político fundamental, pois é na escola que se constrói uma ideologia através da transmissão de valores e crenças, além de ser um ambiente propício para o desenvolvimento de ações educativas em saúde, inclusive, de hábitos alimentares21. Neste contexto, o professor pode ser o principal influenciador de hábitos alimentares saudáveis, pois passa maior parte do tempo com os alunos, conhece suas realidades e tem grande capacidade comunicativa, tornando-se fundamental no processo de formação de hábitos alimentares dos pré-escolares ${ }^{22 .}$

É importante destacar que hábitos e comportamentos começam a ser definidos na infância e, desta forma, são importantes as influências positivas nesta fase. Além disso, se forem transmitidas através de práticas alimentares saudáveis que lhes sejam exemplo, têm maior possibilidade de perdurarem até a vida adulta ${ }^{23}$.

Hábitos são aprendidos a partir da experiência, da observação e da educação. Diante disso, além do papel da família, a equipe da escola é fundamental na alimentação, e na educação alimentar e nutricional das crianças, uma vez que pode oferecer exemplo de hábitos saudáveis ${ }^{24}$.

Algumas pesquisas que investigam fatores associados ao excesso de peso infantil, ainda hoje, centram-se nos determinantes biológicos e no estilo de vida, conferindo menor visibilidade e atenção a aspectos do comportamento alimentar que podem estar envolvidos nesse processo ${ }^{25}$.

O aparecimento de doenças potencialmente evitáveis no período da infância intensifica a fragilidade à saúde por toda a vida, e ao considerar que quase um terço das crianças brasileiras menores de cinco anos estão desprotegidas de segurança, por apresentarem excesso de peso associado, principalmente, ao consumo alimentar inadequado, devese buscar com mais urgência as medidas de proteção das crianças, sobretudo, frente aos impactos em sua atenção à saúde26. Desta forma, ressalta-se a importância de influências positivas na formação de hábitos alimentares na infância, tanto no ambiente familiar, quanto no âmbito escolar.

Vale, ainda, destacar que os demais fatores, como preço, humor, conveniência, familiaridade e aspectos éticos, assumiram menor importância nas escolhas alimentares, neste estudo, apesar de ser claro que o valor dos alimentos influência de forma significativa as escolhas alimentares. Considerando o baixo consumo de frutas, legumes e verduras e 0 aumento do consumo de alimentos processados altamente energéticos e de baixo teor nutritivo na dieta da população urbana do País, estudos indicam que o preço dos alimentos pode representar uma limitação para a adoção de uma alimentação saudável e adequada ${ }^{27}$.

Diferentemente dessa pesquisa, um estudo que avaliou os fatores que influenciam as escolhas alimentares na hora do almoço entre trabalhadores americanos, reportou queas variáveis relacionadas à escolha alimentar para a maioria dos 
indivíduos foi aconveniência (34\%),seguida pelo sabor (28\%) e ocusto (21\%)28. A conveniência engloba alguns critérios como: que seja fácil de preparar, que possa ser cozinhado de forma muito simples, que não leve muito tempo para ser preparado, que possa ser comprado em locais perto de onde as pessoas moram ou trabalham e que seja fácil de achar em mercearias e supermercados ${ }^{16}$.

Desta forma, é importante destacar que, por se tratar de um estudo que busca identificar o quanto os critérios de escolhas dos professores podem influenciar nas escolhas alimentares das crianças durante o período pré-escolar, é necessário dizer que estes fatores, como preço, humor, conveniência, familiaridade e comportamento ético, com menores valores, são influenciadores menos representativos nas escolhas alimentares no ambiente escolar, do que quando comparado aos quatro primeiros. Assim, estes resultados podem ser considerados satisfatórios, na medida em que os fatores atribuídos com maior importância pelos participantes foram relacionados à prática de hábitos alimentares saudáveis, podendo influenciar positivamente nas escolhas alimentares das crianças.

Com relação às informações obtidas através do formulário de marcadores de consumo alimentar, foi possível observar, a partir da frequência absoluta e relativa, que as respostas positivas para o consumo de alimentos marcadores de uma alimentação saudável (alimentos in natura e minimamente processados) no dia anterior ao questionamento, tiveram uma de variação de $67 \%$ a $83 \%$ entre os participantes. Já para alimentos marcadores de um consumo não saudável (alimentos processados e ultraprocessados), a variação foi de $20 \%$ a $36 \%$.

É importante destacar que os dados da Vigilância de Fatores de Risco e Proteção para Doenças Crônicas por Inquérito Telefônico (VIGITEL) mostram que o consumo regular de feijão na população brasileira caiu de 67,5\% em 2012 para 61,3\% em 201610, informação próxima do evidenciado neste estudo (67\%).

Apesar de haver um registro maior quanto ao consumo de alimentos marcadores de uma alimentação saudável, é preciso destacar que o consumo de processados e ultraprocessados tem uma representatividade importante $(20 \%$ a $30 \%)$ de consumo. Esta informação é relevante no ponto em que a VIGITEL registra que o consumo de bebidas adocicadas (sucos artificiais e refrigerante) reduziu de 30,9\% em 2007 para 16,5\% em 201610.

Ao aproximar os resultados provenientes do questionário sobre escolhas alimentares dos obtidos com o formulário de marcadores do consumo alimentar, parece haver uma coerência, na medida em que o grupo dos alimentos in natura e minimamente processadosforam os mais presentes na avaliação de consumo atual, e os fatores de maior representatividade para escolhas alimentares foram apelo sensorial, saúde e conteúdo natural.

Apesar disso, é necessário destacar que os marcadores de um consumo alimentar não saudável são bastante presentes no consumo atual dos participantes da pesquisa, indicando que o consumo deste grupo de alimentos ainda é um desafio para promoção da alimentação adequada e saudável, e que são necessárias ações de educação alimentar e nutricional para que este perfil de consumo, gradativamente, possa ser melhorado, considerando queestes produtos apresentam alta densidade energética, excesso de gorduras totais e saturadas, maiores concentrações de açúcar e/ou sódio e baixo teor de fibras29, contribuindo para a ocorrência dedoenças crônicas não transmissiveis relacionadas à má alimentação30.

Tendo em vista que é na infância que são formados mais intensamente os hábitos alimentares com maiores possibilidades de perdurarem no curso da vida, ressalta-se não somente a responsabilidade da família, mas também a da escola, na mesma dimensão, de promover o aprendizado cotidiano e concreto de práticas alimentares saudáveis. Diante disso, 0 professor é ator fundamental neste processo, uma vez que é o profissional que está diretamente ligado aos mais diversos aprendizados construídos pelos alunos, visto como modelo ideal a ser seguido a partir de suas escolhas alimentares ${ }^{31}$.

\section{Conclusão}

A partir do contexto estudado, pondera-se que os professores podem ser influências positivas na formação de hábitos alimentares de crianças. Porém, ainda é preciso trabalhar com este público de forma a aproximá-lo desta responsabilidade e prepará-lo para a abordagem da alimentação na infância, especialmente em um momento em que se observa um distanciamento familiar no momento das refeições, o que é proporcional ao aumento do tempo de permanência da criança no ambiente escolar.

Além disso, destaca-se a importância de consolidar a escola como ambiente promotor da alimentação adequada e saudável, e o professor como mediador deste processo, pois as práticas alimentares adotadas nos primeiros anos de 
AVALIAÇÃO DOS ASPECTOS RELACIONADOS ÀS ESCOLHAS ALIMENTARES ENTRE PROFESSORES DE CENTROS EDUCACIONAIS INFANTIS

vida são determinantes para a formação de hábitos e comportamentos alimentares saudáveis, que podem se perpetuar pelos demais ciclos da vida e agir de forma progressiva no combate das doenças crônicas não transmissíveis.

\section{Referências}

1.Brasil. Ministério da Saúde. Secretaria de Atenção à Saúde. Departamento de Atenção Básica. Guia alimentar para a população brasileira: promovendo a alimentação saudável. Brasília: Ministério da Saúde, 2008.

2.Jomori MM, Proença RPC, Calvo MCM. Determinantes de escolha alimentar. Rev Nutr. 2008;21(1):63-73.

3.Bercini LO, Masukaw MLT, Martins MR, Labegalini MPC, Alves NB. Alimentação da criança no primeiro ano de vida em Maringá, PR. Cienc Cuid Saude. 2007;6(2):404-10.

4.Soares ACF, Lazzari ACM, Ferdinandi MN. Análise da importância dos conteúdos da disciplina de educação nutricional no ensino fundamental segundo professores de escolas públicas e privadas da cidade de Maringá-Paraná. Rev Saúde Pesqui. 2009;2(2):179-84.

5.Schmitz BAS, Recine E, Cardoso GT, Silva JRM, Amorim NFA, Bernardon R, et al. A escola promovendo hábitos alimentares saudáveis: uma proposta metodológica de capacitação para educadores e donos de cantina escolar. Cad Saúde Pública. 2008;24(2):312-22.

6.Pietruszynski EB, Albiero KA, Pöpper G, Teixeira PF. Práticas pedagógicas envolvendo a alimentação no ambiente escolar: apresentação de uma proposta. Rev Teoria e Prática da Educação. 2010;13(2):223-29.

7.Gomes DM, Bastos KPL, Souza ECG, Paixão JA, Arêdes EM. O papel da escola na formação do bom hábito alimentar. Rev Cien da Faminas. 2005;1(1):28.

8.Souza AM, Pereira RA, Yokoo EM, Levy RB, Sichieri R. Alimentos mais consumidos no Brasil: Inquérito Naาcional de Alimentação 2008-2009. Rev Saúde Pública. 2013;47(1):190-9.

9.Brasil. Ministério da Saúde. Secretaria de Atenção à Saúde. Departamento de Atenção Básica. Guia alimentar para a população brasileira. Brasília: Ministério da Saúde, 2014.

10.Brasil. Ministério da Saúde. Secretaria de Vigilância em Saúde. Departamento de Vigilância de Doenças e Agravos não Transmissíveis e Promoção da Saúde. Vigilância de Fatores de Risco e Proteção para Doenças Crônicas por Inquérito Telefônico. Brasília: Ministério da Saúde, 2016.

11.Brasil. Ministério da Saúde. Sistema de Vigilância Alimentar e Nutricional (SISVAN): Relatórios Públicos do SISVAN. Brasilia: Ministério da Saúde, 2016. Disponível em: .

12.Madruga SW, Araújo CLP, Bertoldi AD, Neutzling MB. Manutenção dos padrões alimentares da infância à adolescência. Rev Saúde Pública. 2012;46(2):376-86,

13.Brasil. Ministério da Saúde. Secretaria de Atenção à Saúde. Departamento de Atenção Básica. Programa Saúde na Escola (PSE). Brasília: Ministério da Saúde, 2011.

14.Bizzo MLG, Leder L. Educação nutricional nos parâmetros curriculares nacionais para o ensino fundamental. Rev Nutr. 2005;18(5):661-67. 
15.Steptoe A, Pollard TM, Wardle J. Development of a measure of the motives underlying the selection of food: the food choice questionnaire. Rev Appetite. 1995;25(3):267-84.

16. Heitor SFD, Estima CCP, Neves FJ, Aguiar AS, Castro SS, Ferreira JES. Tradução e adaptação cultural do questionário sobre motivo das escolhas alimentares (Food Choice Questionnaire - FCQ) para a língua portuguesa. Ciênc Saúde Coletiva. 2015;20(8):2339-46

17.Cohen L, Manion L, Morrison K. Research Methods in Education: RoutledgeFalmer. Teaching in Higher Education. 5. ed. London: Routledge; 2000.

18.Brasil. Ministério da Saúde. Secretaria de Atenção à Saúde. Departamento de Atenção Básica. Orientações para Avaliação de Marcadores de Consumo Alimentar na atenção básica. Brasília: Ministério da Saúde, 2015.

19.Januszewska R, Pieniak Z, Verbeke W. Food choice questionnaire revisited in four countries. Does it still measure the same? Rev Appetite. 2011;57(1):94-8.

20.Guimarães G, Aerts D, Câmara SG. A escola promotora da saúde e o desenvolvimento de habilidades sociais. Rev Diaphora. 2014;12(2):88-95.

21.Mont'alverne DGB, Catrib AMF. Promoção da saúde e as escolas: como avançar. Rev Bras Promoç Saúde. 2013;26(3):307-8.

22.Davanço GM, Taddei JAAC, Gaglianone CP. Conhecimentos, atitudes e práticas de professores de ciclo básico, expostos e não expostos a Curso de Educação Nutricional. Rev Nutr. 2004;17(2):177-84.

23.Santos DLC, Cassimiro TC, Simony RF, Santos MB, Valente MLF, Olim SCV, et al. Avaliação nutricional e estilo de vida de educadoras de uma creche do município de São Paulo. Rev O Mundo da Saúde. 2011;35(4):454-8.

24.Marin T,Berton P,Espírito Santo LKR. Educação nutricional e alimentar: por uma correta formação dos hábitos alimentares. Rev Fapciência.2009;3(7):72-8.

25.Passos DR, Gigante DP, Maciel FV, Matijasevich A. comportamento alimentar infantil: comparação entre crianças sem e com excesso de peso em uma escola do município de Pelotas, RS. Rev Paul Pediatr. 2015;33(1):42-9.

26.Rosaneli CF, Spinelli SMC, Cunha TR. Bioética e infância: a alimentação como referência na atenção à saúde. Rev Iberoam Bioética. 2016;2(1):1-10.

27.Mondini L,Martins VA, Margarido MA, Bueno CRF, Claro RM, Levy RB. Evolução dos preços de alimentos em São Paulo, Brasil, 1980-2009: considerações sobre o acesso à alimentação saudável. Rev Inform Econ.2012;42(2):47-55.

28.Blanck HM, Yaroch AL, Atienza AA, Yi SL, Zhang J, Mâsse LC.Factors influencing lunchtime food choices among working Americans. Health Educ \& Behav. 2007;36(2):289-301.

29.Moubarac JC, Martins AP, Claro RM, Levy RB, Cannon G, Monteiro CA. Consumption of ultra-processd foods and likely impact on human health. Evidence from. Public Health Nutr. 2012;16(12):2240-8.

30.Oliveira GMM, Andrade WM. O preço da obesidade. Rev Bras Cardiol. 2013;26(4):238-40.

31.Gallina LS, Teo CRPA,Szinwelski NK,Bohrz S, Grahl F,Albani G. Hábito alimentar do professor: importante elemento para a promoção da saúde no ambiente escolar. RevSimbio-Logias.2013;6(9):105-16. 